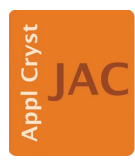

JOURNAL OF

APPLIED

CRYSTALLOGRAPHY

ISSN 1600-5767

Received 24 June 2015

Accepted 26 August 2015

Edited by G. Kostorz, ETH Zurich, Switzerland

Keywords: nanocrystalline metals; catalysts; line profile analysis; surface electrochemistry; transmission electron microscopy.

Supporting information: this article has supporting information at journals.iucr.org/j

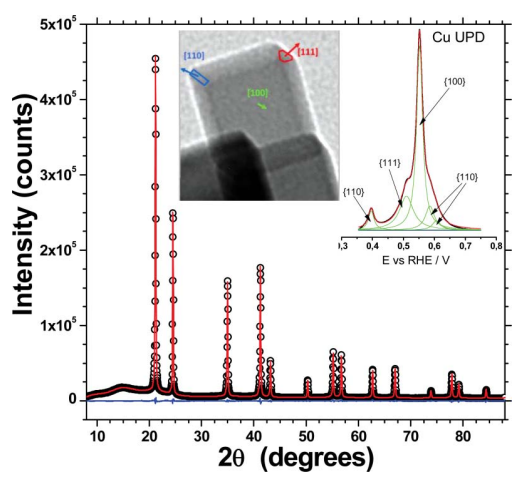

C 2015 International Union of Crystallography

\section{Structure and morphology of shape-controlled Pd nanocrystals}

\author{
Jose Solla-Gullon, ${ }^{a}$ Emmanuel Garnier, ${ }^{\mathrm{a}}$ Juan M. Feliu, ${ }^{\mathrm{a}}$ Matteo Leoni, ${ }^{\mathrm{b}}$ Alberto \\ Leonardi ${ }^{\mathrm{b}}$ and Paolo Scardi ${ }^{\mathrm{b} *}$
}

a Institute of Electrochemistry, University of Alicante, Carretera de San Vicente del Raspeig s/n, Alicante 03690, Spain, and
b Department of Civil, Environmental and Mechanical Engineering, University of Trento, Via Mesiano 77, Trento 38123,
Italy. *Correspondence e-mail: paolo.scardi@unitn.it

Pd nanocrystals were produced with uniform truncated-cube shape and a narrow size distribution, yielding controlled surface area fractions from low Miller index (\{100\}, $\{110\},\{111\})$ crystalline facets. Details on the structure and morphology of the nanocrystals were obtained by combining X-ray powder diffraction line profile analysis, high-resolution transmission electron microscopy and surface electrochemistry based on $\mathrm{Cu}$ underpotential deposition.

\section{Introduction}

Recent progress in nanotechnology has led to the synthesis of size- and shape-controlled metal and metal alloy nanoparticles, which find applications in fields like catalysis, electrocatalysis, plasmonics, sensing and nanomedicine, just to cite a few examples (Sun \& Xia, 2002; Tao et al., 2008; Lee et al., 2009; Xia et al., 2009; Chen \& Holt-Hindle, 2010; Chiu et al., 2011; Gu et al., 2012; Zhang et al., 2013).

Transmission electron microscopy (TEM) is commonly used to observe nanoparticles. Traditional and high-angle annular dark field imaging, as well as three-dimensional tomography, can capture the shape features, whereas, for example, highresolution TEM (HRTEM) and aberration-corrected microscopes coupled with exit wavefunction restoration can unravel local information on the structure of bulk and surfaces (Gontard et al., 2007; Chang et al., 2008, 2010). X-ray powder diffraction (XRD) is frequently used to complement microscopy, providing information on a statistically much larger ensemble of nanoparticles than usually probed by TEM. Most applications of XRD, though, are limited to a qualitative assay (phase identification) or to the estimation of an average crystallite size by the Scherrer equation or by similar approaches exploiting the integral breadth of the line profiles (Klug \& Alexander, 1974; Scardi et al., 2004). More details on the nanocrystals can be obtained by the analysis of the whole powder diffraction pattern, including lattice defects and domain size distribution (e.g. see Scardi, 2008, and references therein), a possibility recently extended to nanocrystals with virtually any shape (Leonardi et al., 2012).

The presence of surfactants or adsorbates, hardly quantifiable by microscopy or by diffraction, can have a dramatic impact on the performance of nanocrystals. Therefore, we propose to extend the combined use of TEM and XRD to surface electrochemistry, as a specific tool to determine the cleanness and relative extent of active nanocrystal surface facets. Such a possibility, fully tested on well oriented singlecrystal metal surfaces (Herrero et al., 2001; Lebedeva et al., 
2002; Solla-Gullón et al., 2008), has also been shown to be very powerful for a precise characterization of the surface structure of a number of shape-controlled metal nanoparticles (Hernández et al., 2004; Vidal-Iglesias et al., 2004; Solla-Gullón et al., 2011). The integration of the three different analytical techniques is especially profitable if one recognizes that nanocrystal shape, in most cases, is not the true goal of the synthesis but just the means to maximize a certain variety of external (catalytically active) surfaces.

\section{Experimental}

\subsection{Specimen preparation}

Pd nanocubes were synthesized using a previously described methodology (Niu et al., 2008; Vidal-Iglesias et al., 2012) in which $\mathrm{H}_{2} \mathrm{PdCl}_{4}$ was reduced with ascorbic acid in an aqueous solution at $368 \mathrm{~K}$ in the presence of cetyltrimethylammonium bromide (CTAB, Aldrich 99\%). To increase the number of particles obtained from a single batch, the synthesis was scaled up by a factor of 20. In detail, $10 \mathrm{ml}$ of $10 \mathrm{mM}$ $\mathrm{H}_{2} \mathrm{PdCl}_{4}$ solution (prepared by dissolving $0.1773 \mathrm{~g}$ of $\mathrm{PdCl}_{2}$ in $10 \mathrm{ml}$ of $0.2 \mathrm{M} \mathrm{HCl}$ and further diluting to $100 \mathrm{ml}$ with ultrapure water) was added to $200 \mathrm{ml}$ of $12.5 \mathrm{mM}$ CTAB solution under stirring, and then the solution was heated at $368 \mathrm{~K}$ for $15 \mathrm{~min}$. Subsequently, $1.6 \mathrm{ml}$ of a freshly prepared $100 \mathrm{mM}$ ascorbic acid solution was added and the reaction was allowed to proceed for $30 \mathrm{~min}$. The surface cleaning of the sample was performed as previously described (Vidal-Iglesias et al., 2012). In brief, the sample was centrifuged twice and redispersed in water. $\mathrm{NaOH}$ was then added (one $\mathrm{NaOH}$ pellet per $50 \mathrm{ml}$ solution). After complete precipitation, the nanoparticles were washed 3-4 times with ultrapure water (Millipore MilliQ) to finally achieve a water suspension. As the quantity of powder was still small, this very same synthesis was repeated three times, and each batch was analysed individually by TEM (see supporting information); subsequently, the powders were mixed together for the XRD and electrochemical measurements.

\subsection{HRTEM characterization}

TEM and HRTEM were employed to investigate the size and shape of the synthesized nanoparticles. TEM experiments were performed with a JEOL JEM-2010 microscope working at $200 \mathrm{kV}$, while HRTEM experiments were carried out on a JEOL 3010 microscope $\left(\mathrm{LaB}_{6}, \mathrm{Cs}=1.1 \mathrm{~mm}\right)$ operated at $300 \mathrm{kV}$, providing a point-to-point resolution of $0.19 \mathrm{~nm}$. The samples for TEM analysis were obtained by placing a droplet of the nanoparticle water suspension (after being twice centrifuged and redispersed in water) onto a formvar/carboncoated copper grid and allowing the solvent to evaporate in air at room temperature. For the HRTEM experiments, the samples were taken from the $\mathrm{NaOH}$-treated aqueous solution (see Specimen preparation for details).

\subsection{XRD analysis}

XRD data were collected at the MCX powder diffraction beamline of the ELETTRA synchrotron radiation facility (Trieste, Italy), using monochromatic radiation with wavelength $\lambda=0.082656 \mathrm{~nm}$. The beamline operates in DebyeScherrer capillary geometry with an optical setup based on two bending mirrors (one of them focusing on the detector), a double-crystal silicon monochromator, and slits to define beam shape and size. Before data collection, accurate alignment was checked by running both a silicon line position standard (NIST SRM 640c) and an $\mathrm{LaB}_{6}$ (NIST SRM 660a) line position and profile standard (Cline et al., 2000); the latter was used to model the instrumental profile according to the traditional Caglioti formula (Caglioti et al., 1958), as implemented in the PM2K software (Leoni et al., 2006; Scardi et al., 2010). XRD data were analysed by the whole powder pattern modelling (WPPM) approach (Scardi \& Leoni, 2002; Scardi, 2008; Scardi et al., 2010) implemented in the PM2K software.

To limit absorption a Kapton capillary of radius $R=$ $0.25 \mathrm{~mm}$ was filled with a low density of Pd nanoparticles. The absorption coefficient, obtained from transmission measurements at different energy, was $\mu=2.71 \mathrm{~cm}^{-1}$, resulting in $\mu R$ well below unity. As a consequence, the XRD data need no absorption corrections. The pattern of an empty capillary was modelled by six broad pseudo-Voigt curves (see supporting information); profiting from the low-absorption condition, this model, multiplied by just one refinable scale parameter, was used as the background in the WPPM analysis of the Pd nanoparticle data.

\subsection{Electrochemical characterization}

The electrochemical characterization of the Pd samples was initially performed in a $0.1 M \mathrm{H}_{2} \mathrm{SO}_{4}$ solution at room temperature in a conventional three-electrode electrochemical cell to evaluate the surface cleanness and surface structure of the Pd nanocubes. Every day an experiment was carried out, new electrolyte solutions were prepared from Millipore Milli-Q water and Suprapur, Merck $96 \% \mathrm{H}_{2} \mathrm{SO}_{4}$, and deaerated with Ar (99.999\%, AlphaGaz). The electrode potential was controlled using an AUTOLAB PGSTAT302N (Metrohm Autolab) system, with a gold wire as counter electrode. The potentials were measured against a reversible hydrogen electrode connected to the cell through a Luggin capillary. For the electrochemical experiments, a droplet (2$10 \mu \mathrm{l})$ of the nanoparticle suspension was pipetted onto a polished glassy carbon substrate $(3 \mathrm{~mm}$ diameter, Goodfellow) and the water was allowed to evaporate in an $\mathrm{Ar}$ atmosphere. Before each experiment, the glassy carbon collector was mechanically polished with alumina and rinsed with ultrapure water to eliminate the nanoparticles from previous experiments. With the aim of maximizing the surface cleanness of the sample, carbon monoxide (CO, 99.997\% AlphaGaz) was adsorbed onto the Pd catalyst at $0.1 \mathrm{~V}$, by bubbling $\mathrm{CO}$ through the electrolyte until complete blockage of the surface, which was monitored by cycling the electrode between 0.1 and $0.35 \mathrm{~V}$. After that, $\mathrm{CO}$ was removed from the 
solution by bubbling $\operatorname{Ar}(10-12$ min Ar per min CO). Finally, the $\mathrm{CO}$ monolayer was electrochemically stripped off from the Pd surface by scanning the potential up to $1.0-1.1 \mathrm{~V}$, and the subsequent voltammogram, corresponding to the CO-free surface, was recorded again. The active surface area of the $\mathrm{Pd}$ nanoparticles was determined by the charge involved in the so-called hydrogen underpotential deposition (UPD) region (between 0.05 and $0.60 \mathrm{~V}$ ) assuming $212 \mu \mathrm{C} \mathrm{cm}^{-2}$ for the total charge, measured in $0.1 \mathrm{M} \mathrm{H}_{2} \mathrm{SO}_{4}$, after the subtraction of the double-layer charging contribution (Woods, 1976). Finally, $\mathrm{Cu}$ UPD experiments were performed in a $0.1 M \mathrm{H}_{2} \mathrm{SO}_{4}+1 \mathrm{~m} M$ $\mathrm{CuSO}_{4}$ (Merck, p.a.) $+1 \mathrm{mM} \mathrm{NaCl}$ (Fluka, p.a.) solution at $50 \mathrm{mV} \mathrm{s}^{-1}$.

\section{Results and discussion}

In general, the TEM data show that most nanoparticles (93\%) have a square or a rectangular footprint, a clear indication of a cuboidal shape (Fig. 1). The facets are planar, albeit in some cases slightly irregular, while the corners and edges are truncated or somehow rounded. Lattice defects are definitely infrequent: stacking faults can be identified in a few nanocrystals, whereas dislocations can only be seen where impacts between nanocrystals have caused plastic deformation. The remaining fraction $(7 \%)$ is made up mostly of multiply twinned (so-called noncrystallographic) nanoparticles like decahedra and icosahedra, but also a few nanorods, flat triangular prisms (nanosheets) and right bipyramids ( $c f$. Table 1). Representative images of all these features are included in the supporting information. A histogram was obtained from 768 cuboids ( 256 per batch), measuring both edges of each nanoparticle. The resulting distribution, including the details for the three starting batches, is shown in Fig. 2(a) (see supporting information for numerical details on

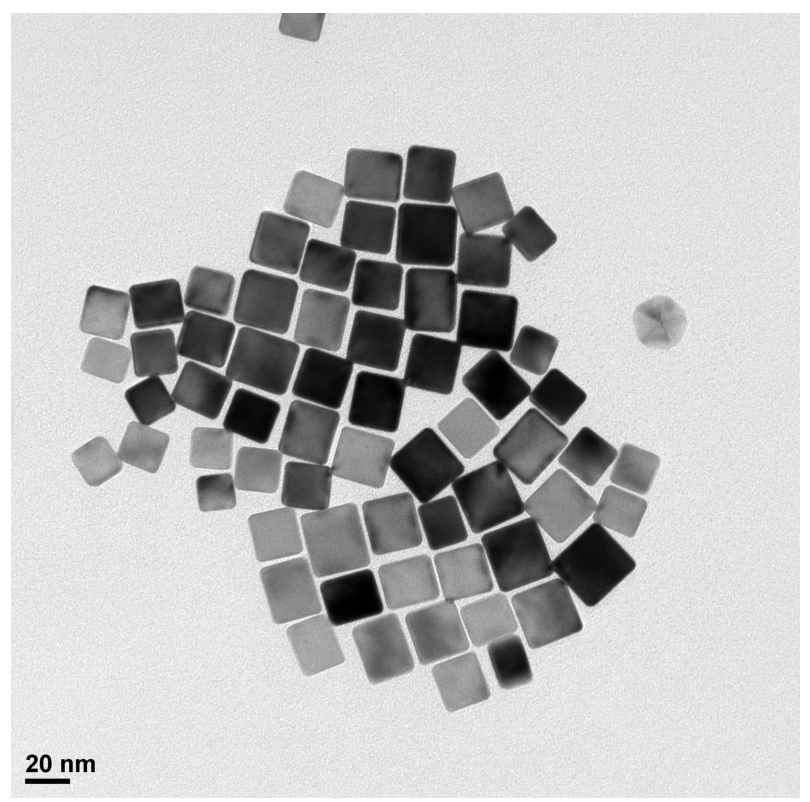

Figure 1

Representative TEM image of the Pd nanocubes used in this work.
Table 1

Statistics of the different Pd nanoparticles.

Details on each separate batch are provided in the supporting information.

\begin{tabular}{|c|c|c|c|c|c|c|}
\hline & Cubes & Nanorods & $\begin{array}{l}\text { Triangular } \\
\text { nanosheet }\end{array}$ & $\begin{array}{l}\text { Right } \\
\text { bipyramids }\end{array}$ & $\begin{array}{l}\text { Icosahedra or } \\
\text { decahedra }\end{array}$ & Undefined \\
\hline Total & 2234 & 14 & 11 & 11 & 121 & 5 \\
\hline$\%$ & 93.24 & 0.58 & 0.46 & 0.46 & 5.05 & 0.21 \\
\hline
\end{tabular}

the three batches and additional TEM images): the main fraction has a mean edge length of 25.7 (1) $\mathrm{nm}$ and a standard deviation of 5.7 (1) $\mathrm{nm}$.

Fig. 3 shows an HRTEM image, collected along the $\langle 110\rangle$ zone axis, of one of the few Pd nanocubes with stacking faults. Despite the apparent rectangular aspect, the object is a cube tilted along one of the $\langle 001\rangle$ axes. Fig. 4 shows a graphical explanation of this phenomenon, quite frequent in the studied powder, while Fig. 5 provides a further example that the observed rectangular footprints are probably the results of particle tilting (see the dark strips on the grains). In those cases, only the shortest edge should be taken into consideration, as it should be more representative of the true size of the

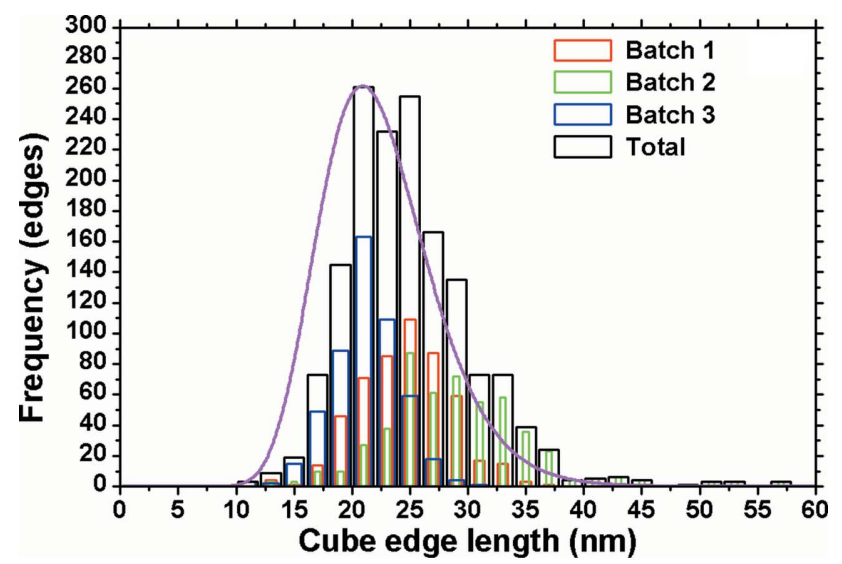

(a)

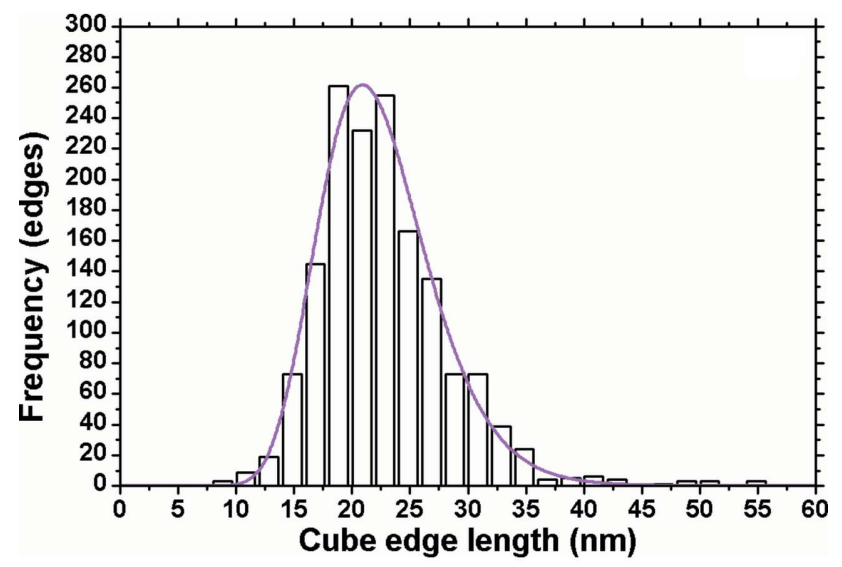

(b)

Figure 2

(a) TEM distribution obtained as the sum of the distributions for the three batches considering the average of the two sides of the features seen in TEM images (bars) versus WPPM result (line). (b) WPPM result (line) versus particle-tilt-corrected TEM distribution (bars). 
domain. Tilting along two directions seems unlikely in the analysed micrographs as a deviation from the rectangular shape was observed in few cases, where cuboidal nanoparticles appear to be standing on a corner (Fig. 5). From the length of the shortest edge we obtain an average cube size of 22.8 (1) nm (cf. Fig. 2b). This is definitely an approximation but is the most plausible one to avoid tilting each particle to determine its true shape and size.

The TEM analysis provides valuable information to build a crystalline domain model for the WPPM analysis. Details on WPPM can be found in the literature (Scardi \& Leoni, 2002; Scardi, 2008; Scardi et al., 2010). In brief, the approach is based on a physical model of the microstructure to generate theoretical expressions for the line profiles. The latter are repre-

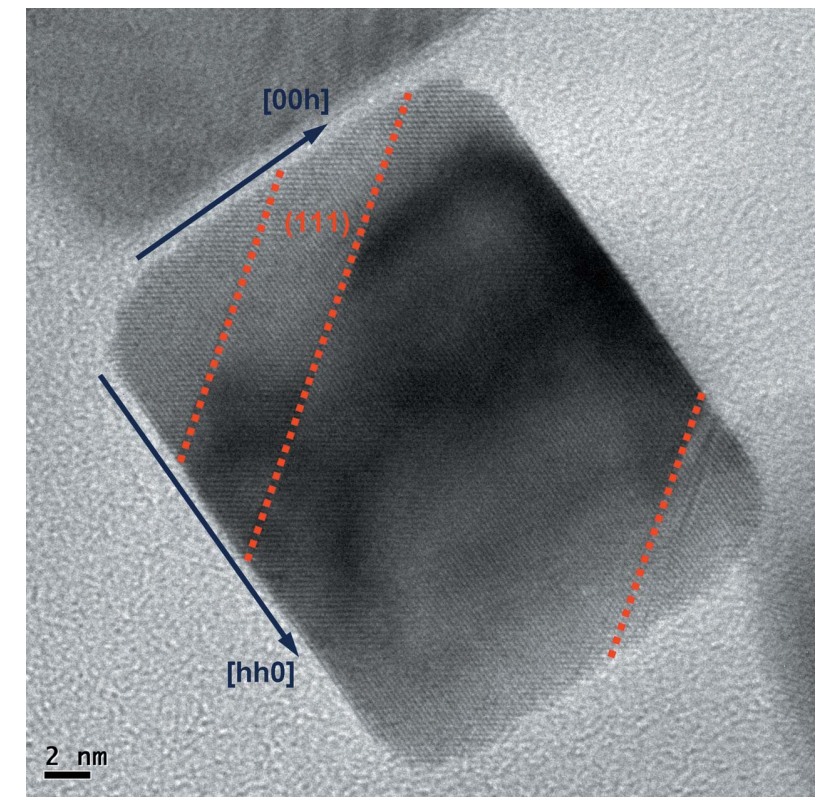

Figure 3

HRTEM image of a single Pd nanocube clearly showing growth defects (stacking faults, red lines), imperfect facets and rounded edges.

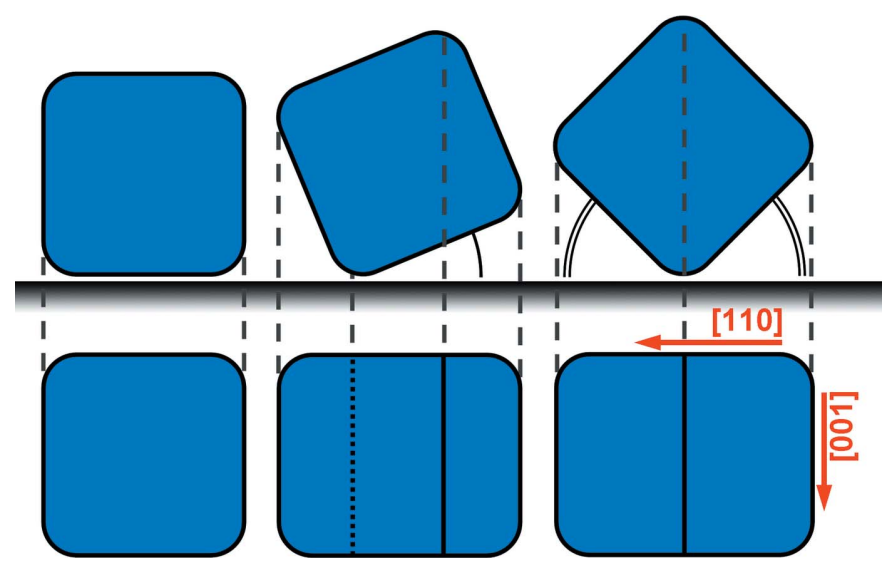

Figure 4

Illustration of the effect of particle tilting on the observed shape of the nanocubes. In the ideal case (cube lying on a face), squares should be observed in TEM images. Rectangles with a maximum of edge length ratio correspond to $45^{\circ}$ tilting. sented as a convolution of profile components produced by all contributing effects, including the microstructure of the material as well as the instrument. Given the instrumental profile from a preliminary analysis of line profile standards ( $c f$. \$2.3), microstructural parameters can be obtained from a nonlinear least-squares fitting of the experimental powder pattern. The convolution problem is conveniently treated by Fourier analysis, requiring the Fourier transforms of the individual profile components.

The main fraction of Pd nanocrystals was represented by cubes with $\{110\}$ truncated edges and triangular $\{111\}$ truncated corners (Fig. 6), assuming a lognormal distribution of edges. The required Fourier transform of the line profile component for the truncated-cube domain shape was obtained from the common volume function (Wilson, 1962) calculated

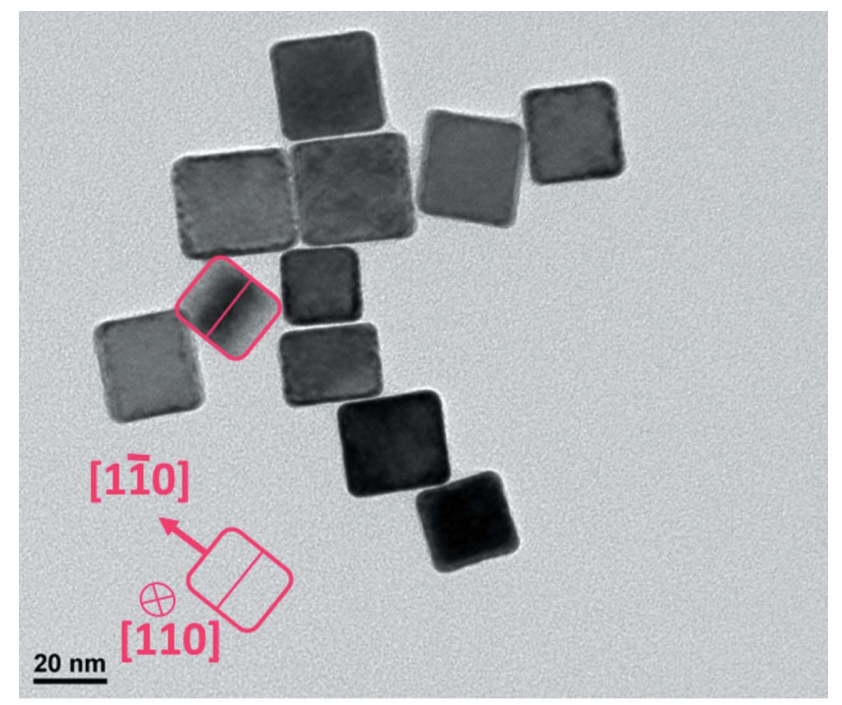

(a)

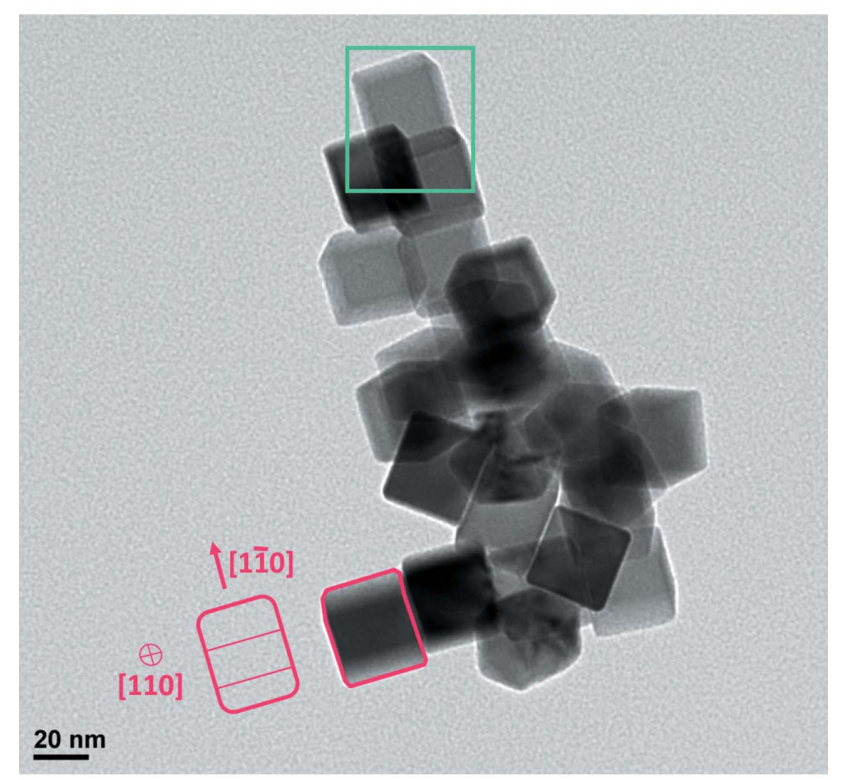

Figure 5

(b)

TEM images containing tilted Pd nanocubes. Rectangular domains showing the clear features of tilting. 
according to an algorithm recently proposed by some of the authors (Leonardi et al., 2012). Corresponding modelling parameters were the lognormal mean $(\mu)$ and variance $(\sigma)$, and a truncation parameter varying from 0 (cube) to 1 (octahedron) as graphically shown in Fig. 7. Simple geometrical considerations provide the surface area fractions of the three different exposed facets as a function of the truncation value. Additional line broadening effects were convolved with the size effect to account for the instrumental profile ( $c f . \S 2.3)$, dislocations and stacking faults, on the basis of the theories of Wilkens and Warren (Scardi \& Leoni, 2002; Scardi, 2008, and references therein). In addition to the unit-cell parameter $\left(a_{0}\right)$, refined parameters were the stacking $(\alpha)$ and twin $(\beta)$ fault probabilities, and the average dislocation density $(\rho)$. The contrast factor was calculated for the edge dislocation case in the primary slip system of face-centred cubic (f.c.c.) Pd, as those were the only visible line defects, while to limit the number of free parameters the effective outer cutoff radius was fixed to the average nanocube size.

Diffraction is sensitive to the volume of the scattering domains, so the contribution of the secondary fraction is quite limited, but it should not be disregarded. A simple and robust model was used for the noncubic (other shapes) fraction, assuming a lognormal distribution of average spherical domains and allowing for the presence of faults according to the Warren theory (Warren, 1990) with corrections to higherorder terms in the solution of the recurrence equations (Estevez-Rams et al., 2008). Noncrystallographic nanoparticles are expected to have a different average unit-cell parameter (usually larger than the bulk) and a quite high inhomogeneous strain as a consequence of the gap between the wedges making up the nanoparticles (Johnson et al., 2008; Gutkin, 2011). Then, in addition to refining a separate unit-cell

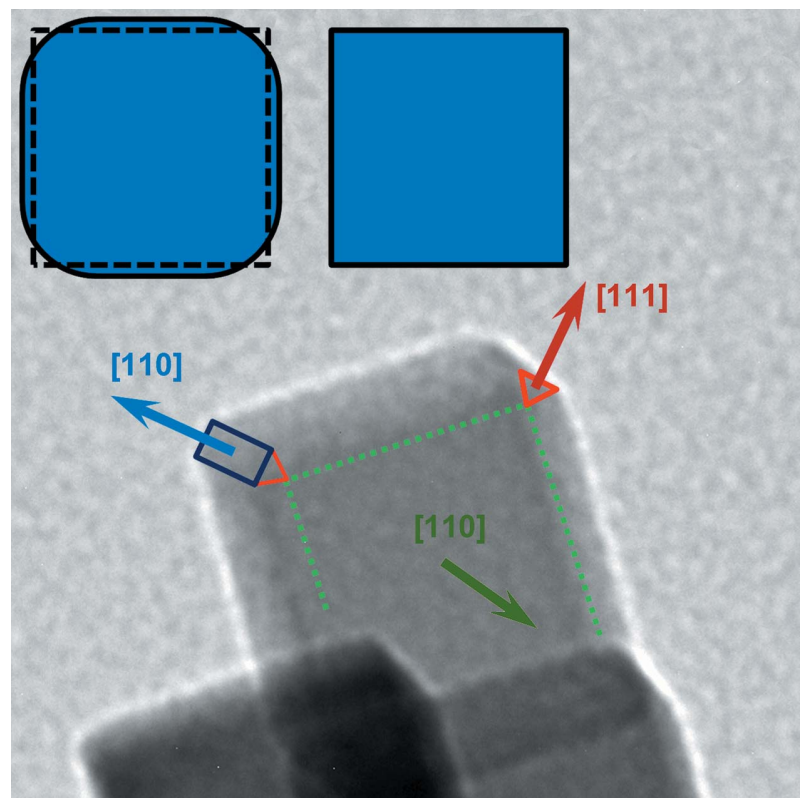

Figure 6

Details of the TEM image in the selected area of Fig. 5(b), showing truncation/rounding of edges and corners. For a given volume, rounded particles are larger than perfect (cubic) particles.
Table 2

Parameters of the main fraction of truncated nanocubes refined via WPPM.

Error estimates (in parentheses) refer to the least significant digit. See the text for details.

\begin{tabular}{llll}
\hline$a_{0}$ & $0.388974(2)$ & $\mathrm{nm}$ & Unit-cell parameter \\
$\rho$ & $3.6(2) \times 10^{13}$ & $\mathrm{~m}^{-2}$ & Average dislocation density \\
$\beta$ & $5(1) \times 10^{-2}$ & $\%$ & Twin fault probability \\
$\mu$ & $3.142(3)$ & - & Lognormal mean \\
$\sigma$ & $0.220(2)$ & - & Lognormal variance \\
Truncation & $0.232(4)$ & - & Truncation fraction parameter \\
\hline
\end{tabular}

parameter for the fraction of other shapes, we also implemented a recently proposed model of microstrain broadening, which already proved to be effective in nanocrystalline metals (Leonardi \& Scardi, 2015; Scardi et al., 2015).

The experimental pattern and the corresponding WPPM refinement are shown in Fig. 8, while the main results are reported in Table 2. The good agreement, witnessed by the flat difference curve and by the good statistical indices $\left(R_{\mathrm{wp}}=\right.$ $\left.2.12 \%, R_{\exp }=0.97 \%\right)$, results in a nanocube edge distribution quite close to the distribution obtained by TEM (Fig. $2 b$ ). WPPM provides an average of $23.7(2) \mathrm{nm}$, a distribution standard deviation of $5.29(5) \mathrm{nm}$ and a truncation factor of 0.232 (4).

The unit-cell parameter is slightly smaller than the literature value for bulk $\mathrm{Pd}(0.38915 \mathrm{~nm})$ because of the surface compression effects often observed in metal nanocrystals (Qi et al., 2009). The refined density of dislocations is quite small, close to the sensitivity level of the technique. The presence of one dislocation per nanocube would give a theoretical density $\rho_{\text {th }}=1 /\langle D\rangle^{2} \simeq 1.8 \times 10^{15} \mathrm{~m}^{-2}$, so that the refined value $[\rho=$ $3.6(2) \times 10^{13} \mathrm{~m}^{-2}$ ] corresponds to one dislocation for about 50 nanocubes, a result compatible with the TEM observations.

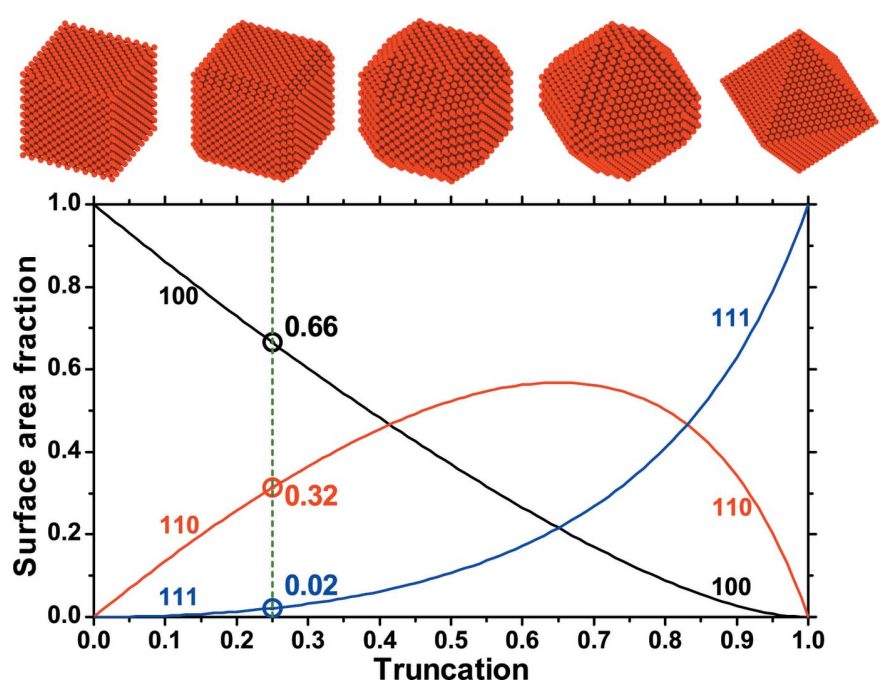

Figure 7

Particle shape model and corresponding relative surface area. The surface area fractions corresponding to the refined truncation are shown: from left to right, truncation 0 (cube), 0.25, 0.5, 0.75 and 1 (octahedron). The dashed line marks the fractions obtained from the XRD modelling (cf. Fig. 8 and corresponding text). 
Stacking faults were found to be twins only. The refined probability $\left[\beta=5(1) \times 10^{-2} \%\right]$ corresponds to an average distance between the twins of $c a 2000$ layers, which would translate into the presence, on average, of a fault every ca 1012 nanocubes. Twin faults are evident in Fig. 3, where more than one planar defect is visible in the same nanocube, and in a few other recorded HRTEM micrographs. Even if a proper statistical evaluation of the faulting distribution cannot be provided by our limited microscopy observations, a quick survey of the available images shows that the refined fault probability is entirely plausible.

Thermal effects were accounted for by implementing a temperature diffuse scattering (TDS) model (Warren, 1990) recently extended to nanosized cube-shaped crystals (Beyerlein et al., 2012), and by the usual Debye-Waller term. The TDS model requires an appropriate scaling between Bragg and diffuse scattering, so that the XRD peak intensities were constrained to the structural model of f.c.c. palladium, as in traditional Rietveld refinements (Rietveld, 1969; Young, 1993; McCusker et al., 1999). As shown in the inset of Fig. 8, the TDS gives a broad signal peaked at the Bragg positions, so that accounting for this diffuse scattering component contributes to improving the accuracy in the measurement of the domain size effects. The refined isotropic Debye-Waller parameter, $B=0.533(2) \AA^{2}$, is close to independent observations on nanocrystalline Pd (Fitzsimmons et al., 1991; Eastman et al., 1992; Scardi et al., 2015). As expected, the value is larger than that for bulk Pd [0.45 (6) $\AA^{2}$; Butt et al., 1988] because of the effect of the loose chemical bonds on the nanocrystal surface, which increase the atomic mean square displacement.

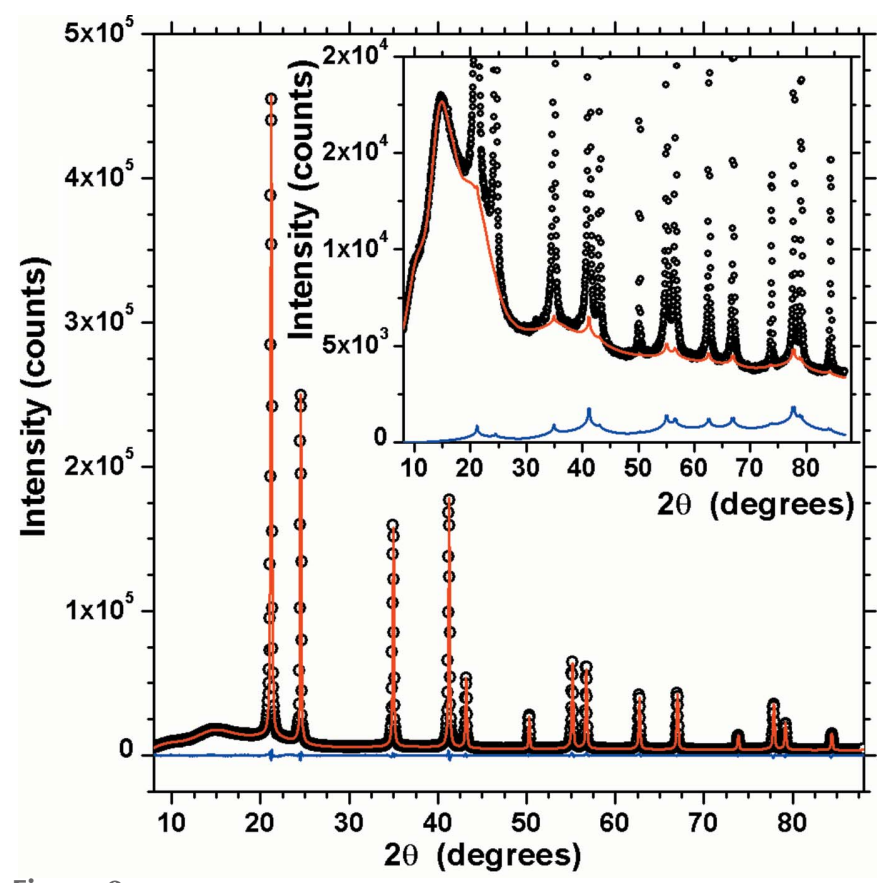

Figure 8

Experimental XRD pattern (circles), WPPM refinement (red line) and difference (blue line). In the inset, the contribution of the Kapton capillary and TDS (line), and TDS alone (line below).
The domain size of the secondary fraction, 5.4 (11) nm with a distribution standard deviation of $3.8(8) \mathrm{nm}$, is roughly equivalent to the size of one of the wedges composing the multiply twinned domains which are the prevailing noncubic objects. At these levels of relative intensity, the peculiar features typical of noncrystallographic particles cannot be fully appreciated/observed. However, a high level of twin faults [6.5 (4)\%] is refined, at the limits of applicability of Warren's model but compatible with the presence of multiply twinned nanoparticles. The microstrain is $\left\langle\varepsilon^{2}\right\rangle^{1 / 2} \simeq 0.003$ (r.m.s. strain averaged over the domain size), and the average unitcell parameter, $a_{0}=0.39040(3) \mathrm{nm}$, is above that of bulk Pd, as also expected for the presence of noncrystallographic nanoparticles, which require considerable non homogeneous (tensile) strain to close the gap between the wedges parallel to the fivefold symmetry axis (Johnson et al., 2008; Gutkin, 2011).

The XRD analysis also provides indirect information on the surface structure of the nanocrystals. As shown in Fig. 7, on the basis of the truncation value refined by the WPPM analysis the distribution of the facet areas of the nanocubes is $66.3,31.5$ and $2.2 \%$, corresponding, respectively, to the $\{100\},\{110\}$ and $\{111\}$ surfaces. However, including the contribution from the noncubic shape fraction ( $\mathrm{ca} 10 \%$ in volume according to XRD), which mostly expose $\{111\}$ and $\{100\}$ facets, the above percentages can be corrected to $c a 64,28$ and $8 \%$ for $\{100\}$, $\{110\}$ and $\{111\}$, respectively.

$\mathrm{Cu}$ UPD experiments were performed on the same $\mathrm{Pd}$ specimen to validate the X-ray diffraction and microscopy results. From previous Pd single-crystal experiments, it is known that this process is sensitive to the surface structure of Pd electrodes (Herrero et al., 2001; Cuesta et al., 1999; VidalIglesias et al., 2006). To illustrate this surface structure sensitivity, Fig. 9 shows the $\mathrm{Cu}$ UPD obtained in a $0.1 \mathrm{M} \mathrm{H}_{2} \mathrm{SO}_{4}+$ $1 \mathrm{mM} \mathrm{CuSO}_{4}+1 \mathrm{mM} \mathrm{NaCl}$ solution with a polyoriented single-crystal Pd bead (Fig. 9a) and with the Pd nanocubes (Fig. 9b). In these plots the potential window is limited to the $\mathrm{Cu}$ UPD region, whereas extended voltammograms including the bulk $\mathrm{Cu}$ deposition and stripping at lower potentials are reported in the supporting information. In the Cu UPD region at the polyoriented Pd bead (Fig. 9a), multiple voltammetric features are observed in the positive going sweep, due to the presence of $\{100\}$ (peak at $0.55 \mathrm{~V}$ ), $\{110\}$ (contributions in the ranges $0.36-0.45$ and $0.58-0.66 \mathrm{~V}$ ) and $\{111\}$ (peak at $0.52 \mathrm{~V}$ ) surface domains, in agreement with previous observations on Pd single-crystal electrode surfaces. The additional contribution observed at $c a 0.32 \mathrm{~V}$ is related to the formation of a second $\mathrm{Cu}$ monolayer on $\{100\}$ surface domains, as previously reported for a Pd(100) single crystal (Cuesta et al., 1999; VidalIglesias et al., 2006). Interestingly, if the surface of a polyoriented Pd bead is disordered by cycling between 0.1 and $1.45 \mathrm{~V}$ to continuously oxidize and reduce its surface [such treatment, previously called 'electrochemical activation', is known to strongly perturb the surface structure, particularly $\{100\}$ and $\{111\}$ terrace domains (Chen et al., 2010)], the characteristic $\mathrm{Cu}$ UPD signatures coming from $\{100\}$ and $\{111\}$ surface domains almost disappear, pointing to a change in the distribution of surface domains. 


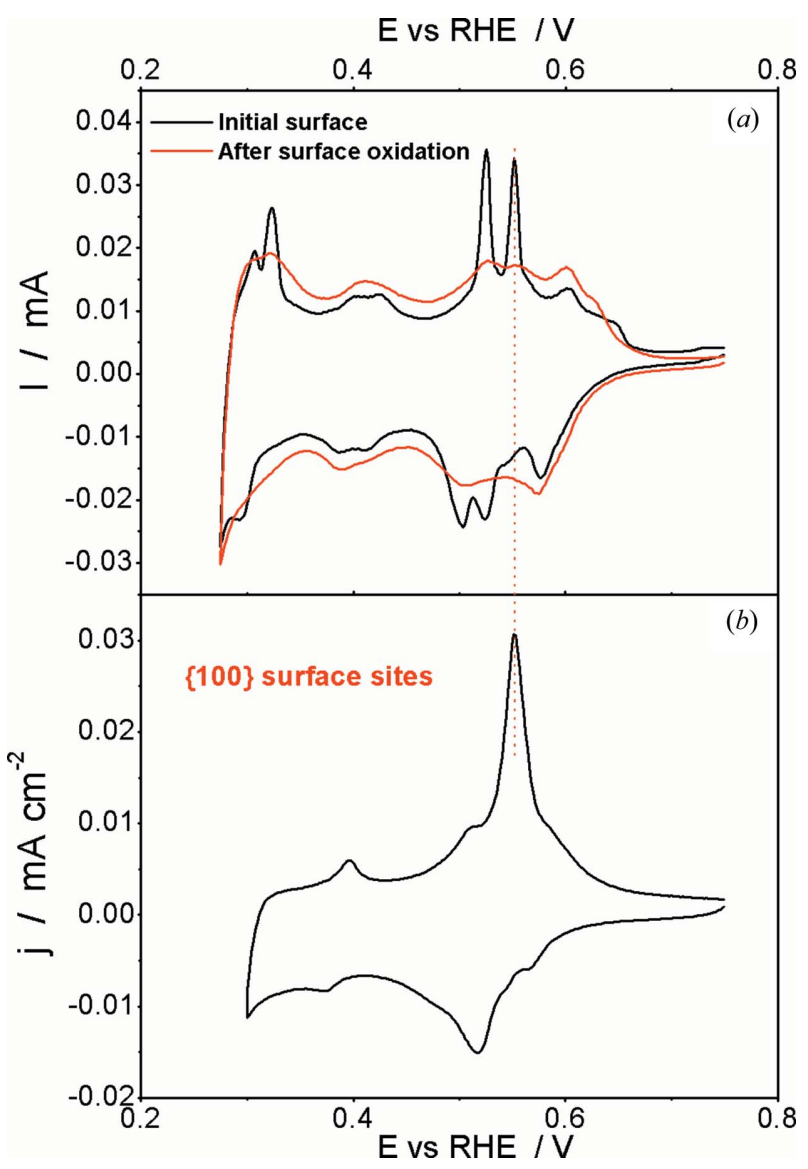

Figure 9

Electrochemical characterization of Pd surfaces. Cu UPD results for $(a)$ polyoriented $\mathrm{Pd}$ bead before and after surface reconstruction and $(b)$ cubic Pd nanoparticles. Test solution $0.1 M \mathrm{H}_{2} \mathrm{SO}_{4}+1 \mathrm{~m} M \mathrm{CuSO}_{4}+$ $1 \mathrm{~m} M \mathrm{NaCl}$, scan rate $50 \mathrm{mV} \mathrm{s}^{-1}$.

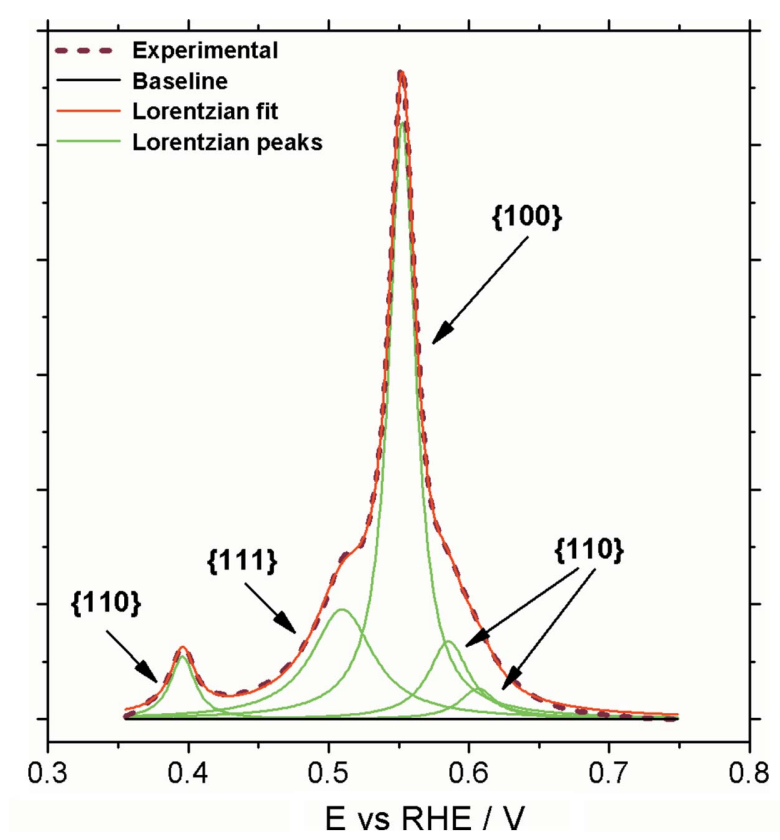

Figure 10

$\mathrm{Cu}$ UPD fitting on the Pd nanocubes.
Fig. 9(b) shows an analogous Cu UPD experiment with the $\mathrm{Pd}$ nanocubes deposited on glassy carbon. Before $\mathrm{Cu}$ UPD experiments, the Pd nanocubes were cleaned as described in \$2.4. The results obtained during this test are summarized in the supporting information. The $\mathrm{Cu}$ UPD profile obtained with $\mathrm{Pd}$ nanocubes shows a main contribution at $0.55 \mathrm{~V}$, characteristic of $\{100\}$ surface domains, while the other peaks appear as minor features. This $\mathrm{Cu}$ UPD behaviour clearly indicates the presence of a preferential $\{100\}$ surface orientation on the Pd nanocubes. Moreover, the well defined $\mathrm{Cu}$ UPD voltammetric response shows the surface cleanness of the nanoparticles.

In order to quantitatively evaluate the percentage of $\{100\}$ surface sites of the cubic Pd nanoparticles, a fitting of the $\mathrm{Cu}$ UPD voltammetric profile was performed using five Lorentzians located at the characteristic contributions of the $\{100\}$, $\{110\}$ and $\{111\}$ surface domains (Fig. 10). The percentage of sites of a given surface is related to the area under its corresponding signal relative to the total area of the $\mathrm{Cu}$ UPD process. This calculation is possible taking into account the calculated charges for a complete $\mathrm{Cu}$ monolayer on $\mathrm{Pd}(111)$, $\operatorname{Pd}(100)$ and $\operatorname{Pd}(110)$ single crystals (486, 421 and $297 \mu \mathrm{C} \mathrm{cm}^{-2}$, respectively) (Cuesta et al., 1999; Vidal-Iglesias et al., 2006) (see supporting information for details). The percentage of $\{100\}$ surface sites on the Pd nanocubes was found to be $57 \pm 3 \%$, slightly lower than the average obtained from the XRD analysis (64\%). This is expected, as the XRD value refers to ideal, although truncated, solids. A closer observation of the HRTEM images shows in fact steps and imperfections on the cube facets as well as rounded corners (Fig. 11 and supporting information), which lower the percentage of $\{100\}$ surface sites and increase the contribution of the other surface sites ( $\{110\}$ and $\{(111\}$ sites). The $\mathrm{Cu}$ UPD analysis is highly sensitive to all surfaces, no matter what their nature (steps, edges, corners, kinks, terraces etc.), all of them contributing to the whole $\mathrm{Cu}$ UPD response. Diffraction is not
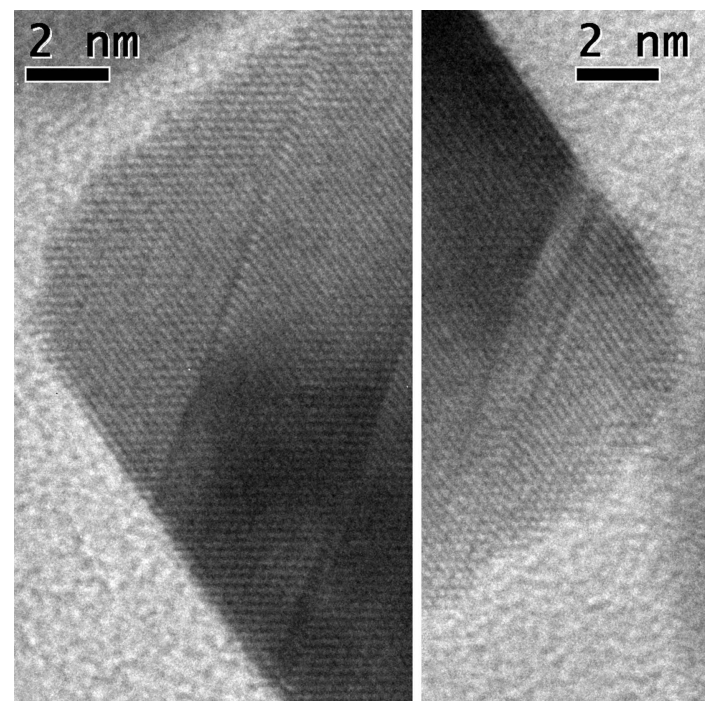

Figure 11

Extracts of an HRTEM micrograph showing irregularities on the surface facets and corners of the Pd nanoparticles. 
sensitive to these features, especially if evenly distributed on all domains, whereas microscopy is able neither to assess the cleanness of the surfaces nor to provide a statistically sound evaluation of the steps to be compared with the voltammetry data. This suggests that for catalytic or electrocatalytic applications, where clean, defined and accessible surface sites are necessary, neither the micrographs nor the diffraction patterns are sufficient to judge the quality of the material: good-looking particles are therefore not necessarily great catalysts, and an appropriate combination of techniques is necessary to understand the real features of the surface of metal nanocrystals.

\section{Related literature}

For additional literature relating to the supporting information, see Maslen (2006), Hoshi et al. (2000, 2002, 2006, 2007), Hara et al. (2007) and Kondo et al. (2009).

\section{Conclusions}

Surface electrochemistry is the ideal complement to TEM and XRD in the characterization of metal nanocrystals. This was demonstrated for a powder of Pd nanocrystals, mostly made of truncated cubes with edge around $22 \mathrm{~nm}$, with a minor fraction of noncrystallographic nanoparticles. TEM showed fine morphological details which were further analysed by a whole powder pattern modelling of the XRD data. Information can be obtained on lattice defects and on domain size and shape, including the average truncation of the nanocubes, which provides a good assessment of the corresponding fractions of $\{100\},\{110\}$ and $\{111\}$ exposed facets. This picture matches the independent results of $\mathrm{Cu}$ underpotential deposition, which is highly sensitive to the surface structure of Pd. Besides explaining fine details of the nanocrystal surface faceting, $\mathrm{Cu}$ UPD provides additional information on the cleanness of the metal surfaces, which is of main interest in applications to catalysis.

\section{Acknowledgements}

ML acknowledges support from the Italian government (Ministero dell'Istruzione, dell'Università e della Ricerca) through the project FIRB Futuro in Ricerca RBFR10CWDA. JMF acknowledges financial support from the MINECO (Spain) project CTQ2013-44083-P and Generalitat Valenciana project PROMETEOII/2014/013. Elettra-Sincrotrone Trieste S.C.p.A is acknowledged for providing the beamtime; the authors are especially grateful to L. Gelisio, C. L. Azanza Ricardo and M. Abdellatief for assistance during the XRD measurements at the MCX beamline.

\section{References}

Beyerlein, K. R., Leoni, M. \& Scardi, P. (2012). Acta Cryst. A68, 382392.

Butt, N. M., Bashir, J., Willis, B. T. M. \& Heger, G. (1988). Acta Cryst. A44, 396-399.

Caglioti, G., Paoletti, A. \& Ricci, F. (1958). Nucl. Instrum. 3, 223-228.

Chang, L., Barnard, A., Gontard, L. \& Dunin-Borkowski, R. (2010). Nano Lett. 10, 3073-3076.
Chang, L., Maunders, C., Baranova, E., Bock, C. \& Botton, G. (2008). Microsc. Microanal. 14, 426-427.

Chen, A. \& Holt-Hindle, P. (2010). Chem. Rev. 110, 3767-3804.

Chen, Q.-S., Solla-Gullón, J., Sun, S.-G. \& Feliu, J. M. (2010). Electrochim. Acta, 55, 7982-7994.

Chiu, C.-Y., Li, Y., Ruan, L., Ye, X., Murray, C. \& Huang, Y. (2011). Nat. Chem. 3, 393-399.

Cline, J. P., Deslattes, R. D., Staudenmann, J.-L., Kessler, E. G., Hudson, L. T., Henins, A. \& Cheary, R. W. (2000). Certificates SRM 640c and SRM 660a. NIST, Gaithersburg, MD, USA.

Cuesta, A., Kibler, L. \& Kolb, D. (1999). J. Electroanal. Chem. 466, 165-168.

Eastman, J., Fitzsimmons, M., Thompson, L., Lawson, A. \& Robinson, R. (1992). Nanostruct. Mater. 1, 465-470.

Estevez-Rams, E., Welzel, U., Pentón Madrigal, A. \& Mittemeijer, E. J. (2008). Acta Cryst. A64, 537-548.

Fitzsimmons, M., Eastman, J., Müller-Stach, M. \& Wallner, G. (1991). Phys. Rev. B, 44, 2452-2460.

Gontard, L., Chang, L.-Y., Hetherington, C., Kirkland, A., Ozkaya, D. \& Dunin-Borkowski, R. (2007). Angew. Chem. Int. Ed. 46, 3683-3685.

Gu, J., Zhang, Y.-W. \& Tao, F. (2012). Chem. Soc. Rev. 41, 8050-8065.

Gutkin, M. Y. (2011). Nanostructured Metals and Alloys: Processing, Microstructure, Mechanical Properties and Applications, ch. 12, pp. 329-374. Cambridge: Woodhead.

Hara, M., Linke, U. \& Wandlowski, T. (2007). Electrochim. Acta, 52, 5733-5748.

Hernández, J., Solla-Gullón, J. \& Herrero, E. (2004). J. Electroanal. Chem. 574, 185-196.

Herrero, E., Buller, L. \& Abruña, H. (2001). Chem. Rev. 101, $1897-$ 1930.

Hoshi, N., Kagaya, K. \& Hori, Y. (2000). J. Electroanal. Chem. 485, $55-60$.

Hoshi, N., Kida, K., Nakamura, M., Nakada, M. \& Osada, K. (2006). J. Phys. Chem. B, 110, 12480- 12484.

Hoshi, N., Kuroda, M. \& Hori, Y. (2002). J. Electroanal. Chem. 521, 155-160.

Hoshi, N., Nakamura, M. \& Kida, K. (2007). Electrochem. Commun. 9, 279-282.

Johnson, C. L., Snoeck, E., Ezcurdia, M., Rodríguez-González, B., Pastoriza-Santos, I., Liz-Marzán, L. M. \& Hÿtch, M. J. (2008). Nat. Mater. 7, 120-124.

Klug, H. P. \& Alexander, L. E. (1974). X-ray Diffraction Procedures, 2nd ed. New York: John Wiley and Sons.

Kondo, S., Nakamura, M., Maki, N. \& Hoshi, N. (2009). J. Phys. Chem. C, 113, 12625-12628.

Lebedeva, N., Koper, M., Feliu, J. \& van Santen, R. (2002). J. Phys. Chem. B, 106, 12938-12947.

Lee, I., Delbecq, F., Morales, R., Albiter, M. \& Zaera, F. (2009). Nat. Mater. 8, 132-138.

Leonardi, A., Leoni, M., Siboni, S. \& Scardi, P. (2012). J. Appl. Cryst. 45, 1162-1172.

Leonardi, A. \& Scardi, P. (2015). Met. Mater. Trans. A. doi:10.1007/ s11661-015-2863-y.

Leoni, M., Confente, T. \& Scardi, P. (2006). Z. Kristallogr. Suppl. 2006, 249-254.

Maslen, E. N. (2006). International Tables for Crystallography, Vol. C, Mathematical, Physical and Chemical Tables,1st online ed., edited by E. Prince, ch. 6.3. Chester: International Union of Crystallography.

McCusker, L. B., Von Dreele, R. B., Cox, D. E., Louër, D. \& Scardi, P. (1999). J. Appl. Cryst. 32, 36-50.

Niu, W., Li, Z.-Y., Shi, L., Liu, X., Li, H., Han, S., Chen, J. \& Xu, G. (2008). Cryst. Growth Des. 8, 4440-4444.

Qi, W., Huang, B. \& Wang, M. (2009). Nanoscale Res. Lett. 4, 269-273.

Rietveld, H. M. (1969). J. Appl. Cryst. 2, 65-71.

Scardi, P. (2008). Powder Diffraction: Theory and Practice, edited by R. E. Dinnebier \& S. J. L. Billinge, ch. 13, pp. 376-413. Cambridge: The Royal Chemistry Society. 
Scardi, P., Leonardi, A., Gelisio, L., Suchomel, M. R., Sneed, B. T., Sheehan, M. K. \& Tsung, C.-K. (2015). Phys. Rev. B, 91, 155414.

Scardi, P. \& Leoni, M. (2002). Acta Cryst. A58, 190-200.

Scardi, P., Leoni, M. \& Delhez, R. (2004). J. Appl. Cryst. 37, 381390.

Scardi, P., Ortolani, M. \& Leoni, M. (2010). Mater. Sci. Forum, 651, 155-171.

Solla-Gullón, J., Rodríguez, P., Herrero, E., Aldaz, A. \& Feliu, J. (2008). Phys. Chem. Chem. Phys. 10, 1359-1373.

Solla-Gullón, J., Vidal-Iglesias, F. \& Feliu, J. (2011). Annu. Rep. Prog. Chem. Sect. C Phys. Chem. 107, 263-297.

Sun, Y. \& Xia, Y. (2002). Science, 298, 2176-2179.

Tao, A., Habas, S. \& Yang, P. (2008). Small, 4, 310-325.

Vidal-Iglesias, F., Al-Akl, A., Watson, D. \& Attard, G. (2006). Electrochem. Commun. 8, 1147-1150.
Vidal-Iglesias, F., Arán-Ais, R., Solla-Gullón, J., Garnier, E., Herrero, E., Aldaz, A. \& Feliu, J. (2012). Phys. Chem. Chem. Phys. 14, 10258-10265.

Vidal-Iglesias, F., Solla-Gullón, J., Rodríguez, P., Herrero, E., Montiel, V., Feliu, J. \& Aldaz, A. (2004). Electrochem. Commun. 6, 10801084.

Warren, B. E. (1990). X-ray Diffraction. New York: Dover.

Wilson, A. J. C. (1962). X-ray Optics: the Diffraction of X-rays by Finite and Imperfect Crystals. London: Methuen.

Woods, R. (1976). Electroanalytical Chemistry: a Series of Advances, Vol. 9, ch. 1. New York: Marcel Dekker.

Xia, Y., Xiong, Y., Lim, B. \& Skrabalak, S. (2009). Angew. Chem. Int. Ed. 48, 60-103.

Young, R. A. (1993). The Rietveld Method. Oxford University Press.

Zhang, H., Jin, M., Xiong, Y., Lim, B. \& Xia, Y. (2013). Acc. Chem. Res. 46, 1783-1794. 